\title{
Ripples in a Pond: Forecasting Industrial Crises
}

\author{
Paul Slovic \\ Decision Research, Inc. \\ 1201 Oak Street \\ Eugene, Oregon 97401
}

\section{Abstract}

Accidents, discoveries of pollution, safety violations, product tampering episodes and other "unfortunate events" occurring within complex industrial systems can be conceptualised as pebbles dropped in a pond. The impacts of such events ripple outward, encompassing first the directly affected victims and property, then the responsible company or agency, and-in the extreme-engulfing other companies, agencies, and industries. Some events make only small ripples; others make large ones, often labelled "industrial crises". This paper explores characteristics of unfortunate events and the ways they are managed that affect the breadth and seriousness of the resulting impacts. In doing so, we point toward the development of models that may help companies forecast and avoid industrial crises.

Complex industrial systems inevitably give rise to "unfortunate events" which vary in type (e.g., accidents, discoveries of pollution, safety violations, product tampering episodes, etc.) and severity. Cost/benefit and risk/benefit analyses typically assess the impacts of such events in terms of direct harm to victims (e.g. deaths, injuries, and damages). Methods exist for attaching probabilities to these direct effects and translating them into monetary costs or disutility indices (Mishan, 1971; Raiffa, 1968; Wilson \& Crouch, 1982).

The impacts of an unfortunate event, however, sometimes extend far beyond these direct harmful effects, and may include significant indirect costs (both monetary and nonmonetary) to the responsible private company or government agency that far exceed direct costs. In some cases, all companies in an industry are affected, regardless of which company was responsible for the mishap. In truly extreme cases, the indirect costs of a mishap may even extend past industry boundaries, affecting companies, industries, and agencies whose business is minimally related to the initial event. Thus, an unfortunate event can be thought of as a pebble dropped in a pond. The ripples extend in concentric circles, encompassing first the directly affected victims, then the responsible company or agency, and, in the extreme, engulfing other companies, agencies, and industries. Some events make only small ripples; others make larger ones, often labeled "industrial crises." This paper explores how characteristics associated with an unfortunate event and the way it is managed act upon perceptions of risk in ways that affect the breadth and seriousness of those impacts. In doing so, we point toward the 
development of models that may help companies and agencies forecast and avoid industrial crises.

\section{Early Theories of Impact}

Early theories linked the seriousness and magnitude of impact of accidents to the number of people killed or injured, or to the amount of property damaged (Ferreira \& Slesin, 1976; Griesmeyer \& Okrent, 1981; Wilson, 1975). However, the accident at the Three Mile Island (TMI) nuclear reactor in 1979 provided a dramatic demonstration that factors besides injury, death, and property damage impose serious costs. Despite the fact that not a single person died at TMI and few if any latent cancer fatalities are expected (President's Commission, 1979), no other accident in our history has produced such costly societal impacts. The accident at TMI devastated the utility that owned and operated the plant. It also imposed enormous costs (estimated at $\$ 500$ billion by one source) on the nuclear industry and on society, through stricter regulation (resulting in increased construction and operating costs), reduced operation of reactors worldwide, greater public opposition to nuclear power, and reliance on more expensive energy sources (EPRI Journal, 1980; Evans \& Hope, 1984; Heising \& George, 1986). It may even have led to a more hostile view of other complex technologies, such as chemical manufacturing and genetic engineering. The point is that traditional economic and risk analyses tend to neglect these higher-order impacts, hence they greatly underestimate the costs associated with certain kinds of mishaps.

Although reaction to the TMl accident was extreme, it is by no means an isolated example. Other recent events that have had enormous indirect impacts include the chemical manufacturing accident at Bhopal, India; accidents resulting from the defective fuel tank of the Ford Pinto; the discovery of pollution from chemical wastes at Love Canal, New York and Times Beach, Missouri; the Tylenol tamperings; the disastrous launch of the space shuttle Challenger; and the reactor meltdown at Chernobyl. Following these extreme events are a myriad of lesser events varying in the breadth and magnitude of their impacts.

New theories and methods of analysis are needed to forecast costly ripple effects so that they may be factored into risk-management decisions. Concepts and methods derived from the study of perceived risk form a first step toward developing a model for understanding and predicting these sorts of impacts.

\section{Perceived Risk and the Psychometric Paradigm}

One approach to assessing the higher order costs of risk-related events has evolved from research on risk perception during the past decade. This work has employed psychometric scaling techniques along with multivariate methods such as factor analysis to produce quantitative representations of people's risk attitudes and perceptions (Slovic, 1987; Slovic, Fischhoff, \& 
Lichtenstein, 1985). This same methodological approach may be able to produce a more adequate model of higher-order impacts.

Within the psychometric paradigm, researchers have asked experts and lay people to judge the riskiness of diverse sets of hazardous activities, substances, and technologies and to judge the need for regulation of these hazards. These judgements have then been related to other judgments about the hazard's status on various characteristics of risk, such as the degree to which exposure to the hazard is voluntary or controllable, whether its consequences evoke feelings of dread, whether its effects can be catastrophic or passed on to future generations; whether its risks are known to science or to those exposed to them, and so on.

Many of these risk characteristics are highly correlated with each other, across the set of hazards. For example, hazards rated as "voluntary" tend also to be rated as "controllable" and "well known"; hazards that are judged to threaten future generations tend also to be seen as having catastrophic potential, and so on. Subjecting these correlations to factor analysis has shown that a rather large set of characteristics can be condensed into a small set of higher-order characteristics or factors, as shown in Figures 1 and 2.

We have found that lay people's risk perceptions and attitudes are closely related to the position of a hazard within this sort of factor space. Specifically, the higher a hazard's score on the factor "Dread Risk" (e.g., the further it is to the right of the space), the higher is its perceived risk, the more people want to see its current risks reduced, and the more they want to see strict regulation employed to achieve the desired reduction in risk (Figure 3).

\section{Accidents as Signals}

An important concept that has emerged from risk-perception research is the notion that the societal cost of an accident or mishap is determined, in part, by what that event signals or portends (Slovic, Lichtenstein, \& Fischhoff, 1984). The informativeness or signal potential of a mishap, and thus its potential social impact, appears to be systematically related to the characteristics of the risk and thus to the location of the event within the factor space (see Figure 4). An accident that takes many lives may produce relatively little social disturbance (beyond that caused the victims' families and friends) if it occurs as part of a familiar and well understood system (e.g. a train wreck). However, a small accident in an unfamiliar system (or one perceived as poorly understood), such as a nuclear reactor, space vehicle, or a recombinant DNA-laboratory, may have immense social consequences if it is perceived as a harbinger of further and possibly catastrophic mishaps.

The concept of accidents as signals was eloquently expressed in an editorial addressing the tragic accident in Bhopal, India: "What truly grips us in these accounts [of disaster] is not so much the numbers as the spectacle of suddenly vanishing competence, of men utterly routed by technology, of fail-safe systems failing with a logic as inexorable as it was once-indeed, 


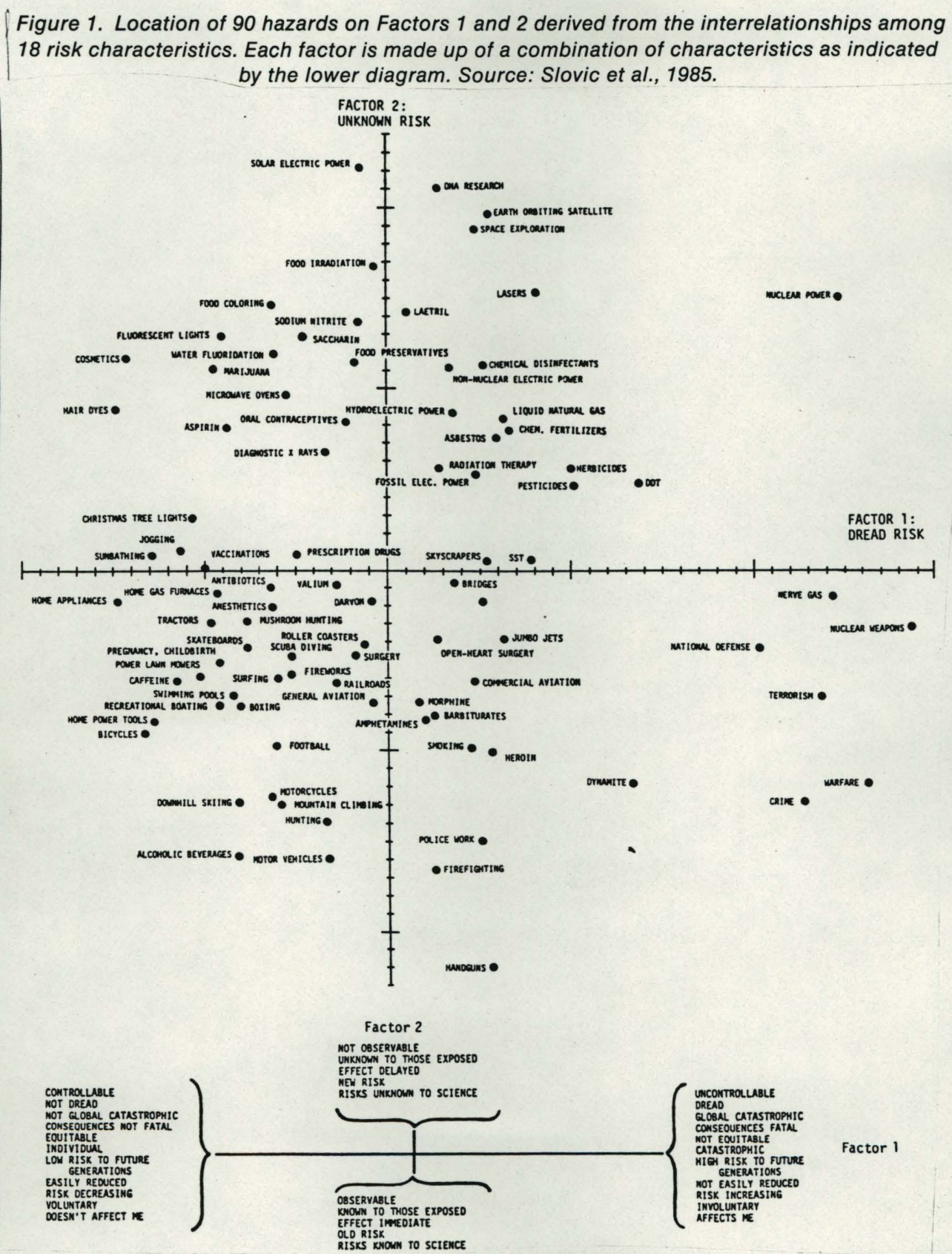

right up until that very moment-unforseeable. And the spectacle haunts us because it seems to carry allegorical import, like the whispery omen of a hovering future" (The New Yorker; February 18, 1985).

The concept of accidents as signals helps explain society's relatively 
Figure 2. Location of 81 hazards on Factors 1 and 2 derived from the interrelationships among 18 risk characteristics. Each factor is made up of a combination of characteristics, as indicated by the lower diagram. Source: Slovic, Fischhoff, and Lichtenstein, 1985.

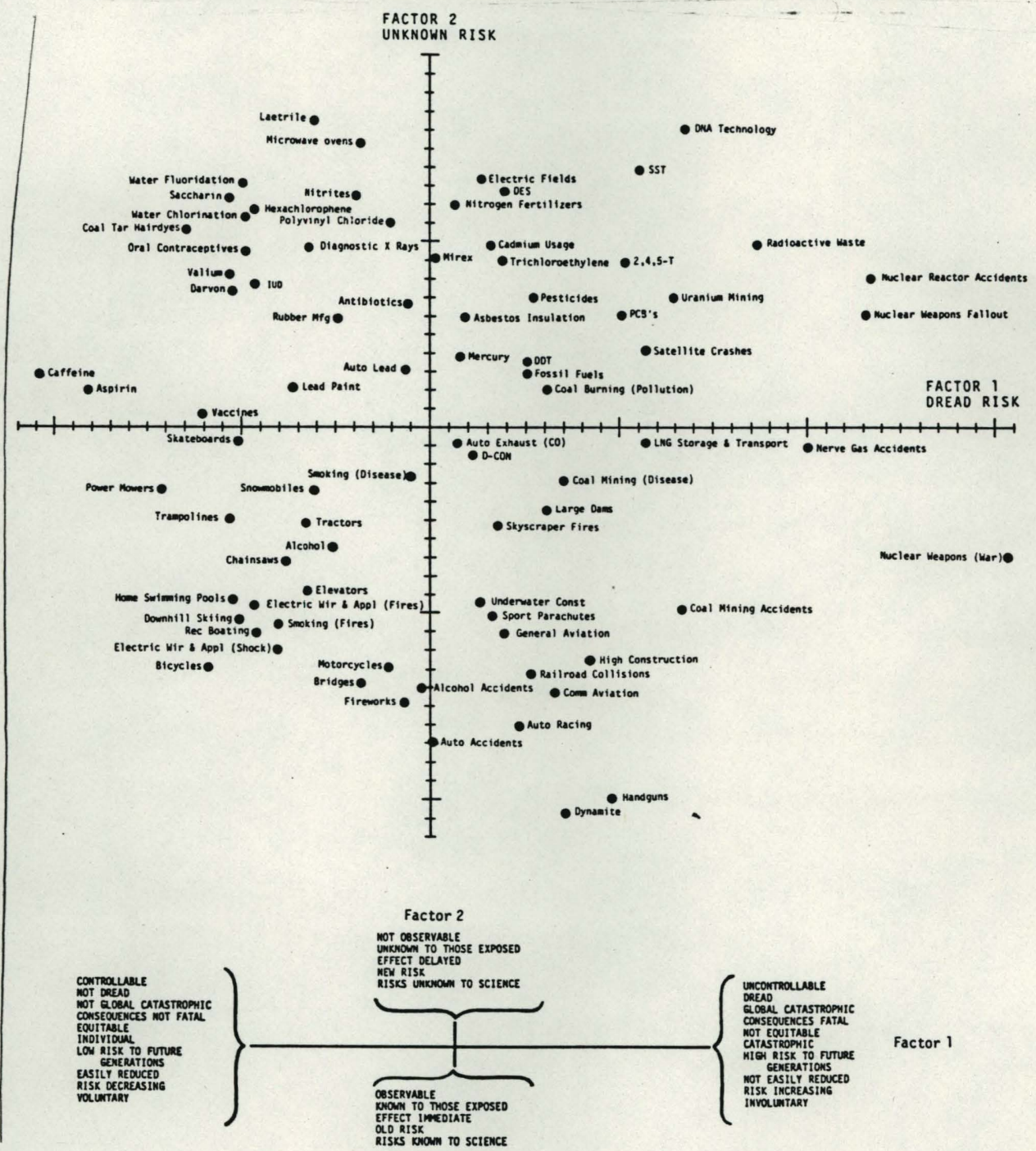

strong response to nuclear power mishaps and, in particular, the TMI accident. Because reactor risks are characterized as poorly understood and catastrophic, accidents may be seen as omens of disaster, thus producing ripple effects resulting in great costs to the industry and society. One implication of this is that effort and expense beyond that indicated by a cost/benefit analysis might be warranted to reduce the possibility of small but frightening reactor accidents. A more general implication of the signal conception 
Figure 3. Attitudes toward regulation of the hazards in Figure 2. The larger the point, the greater the desire for strict regulation to reduce risk.

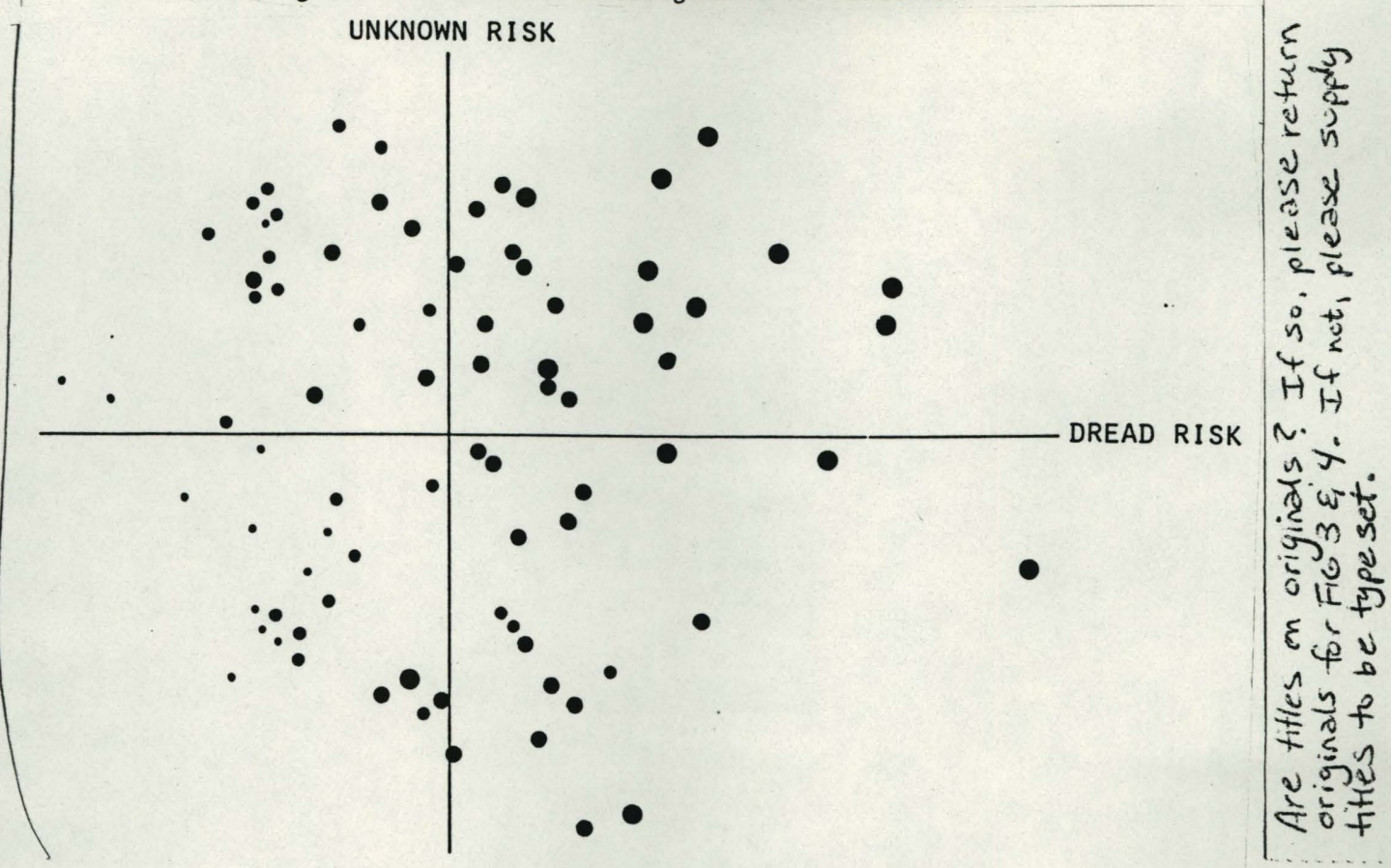

is that any unfortunate events involving hazards that are perceived as poorly understood and catastrophic have the potential to produce large ripples.

This same type of analysis has also been applied to diverse hazard scenarios within a single technological domain such as railroad transport (Kraus \& Slovic, in press) or automobiles (Slovic, MacGregor, \& Kraus, 1987). Kraus and Slovic constructed 49 railroad hazard scenarios varying with respect to type of train, type of cargo, location of the accident, and the nature and cause of the accident (e.g., "A high-speed train carrying passengers through a mountain tunnel derails due to a mechanical system failure"). The results showed that these railroad hazards were highly differentiated, just like the hazards in Figures 1 and 2. The highest signal potential (and thus the highest potential for large ripple effects) was associated with accidents involving trains carrying hazardous chemicals.

The study by Slovic, MacGregor and Kraus examined perceptions of risk and signal value for 40 structural defects in automobiles. Multivariate analysis of these defects, which were rated in terms of various characteristics of risk, predicted judgments of riskiness and signal value quite well. One defect stood out in this analysis, much as nuclear power has stood out in previous studies. It was a fuel tank rupture upon impact, creating the possibility of a fire and burn injuries. This, of course, is the infamous design problem that 
Figure 4. Relation between signal potential and risk characterization for 30 hazards in Figure 2. The larger the point, the greater the degree to which an accident involving that hazard was judged to "serve as a warning signal for society, providing new information about the probability that similar or even more destructive mishaps might occur within this type of activity." The higher-order costs of a mishap are likely to be correlated with signal potential.

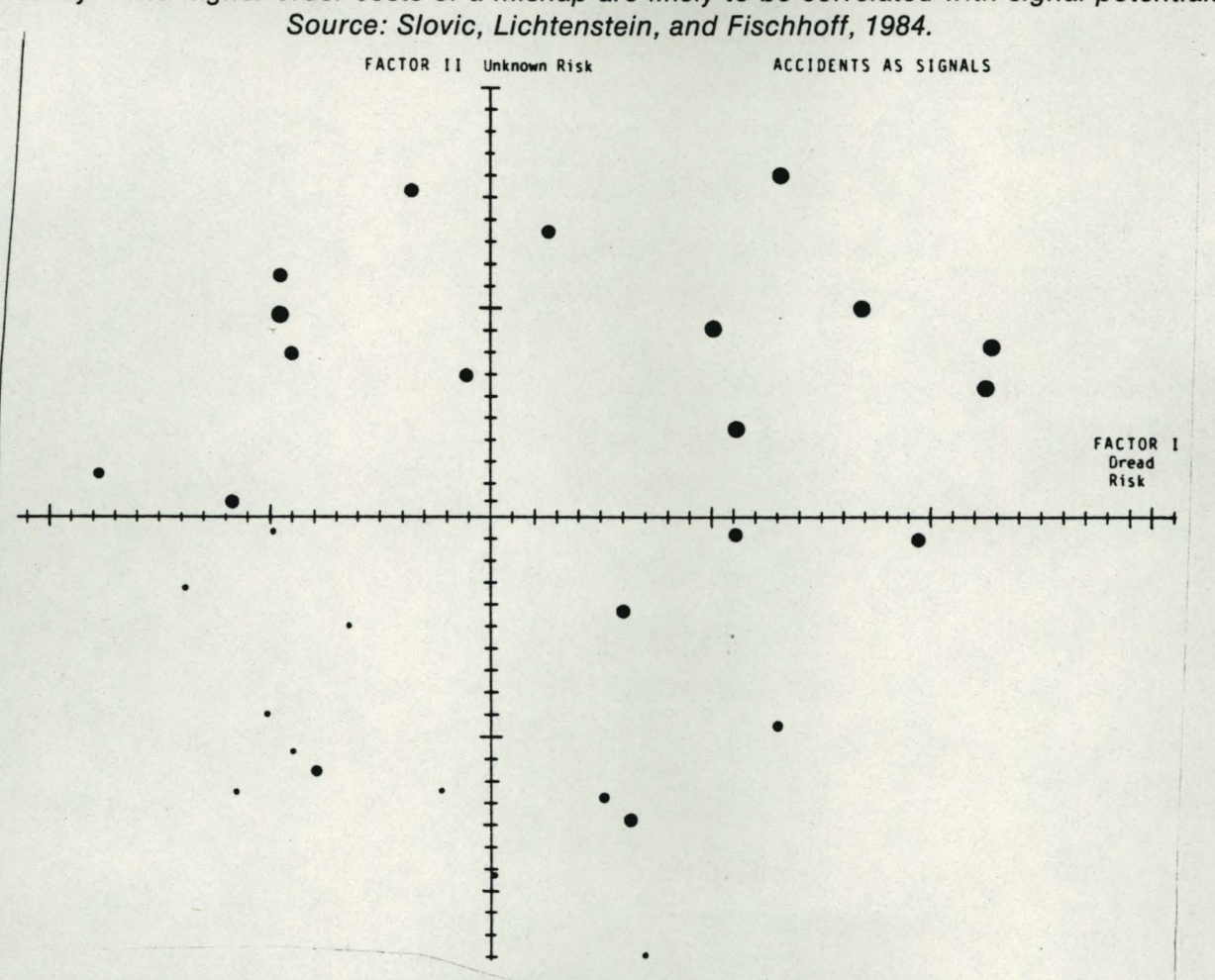

plagued the Ford Pinto and that Ford had allegedly declined to correct because a cost/benefit analysis indicated that the costs greatly exceeded the expected benefits from increased safety. Had Ford done a psychometric analysis such as ours, the analysis might have highlighted this particular defect as one whose seriousness and higher-order costs (e.g. lawsuits, damage to the company's reputation) were likely to be greatly underestimated by their cost/benefit analysis.

\section{Research Directions}

We are presently conducting research along two fronts, theoretical and empirical, in order to improve our ability to forecast costly ripple effects. On the theoretical side we are attempting to develop a much broader view of the negative impacts that might result from unfortunate events and to cast a wider net to uncover characteristics that might predict the seriousness of those impacts. The theoretical framework that we develop will then be used to design a program of empirical research.

Figure 5 illustrates some key elements of a theoretical analysis of im- 
Figure 5. A model of impact for unfortunate events.

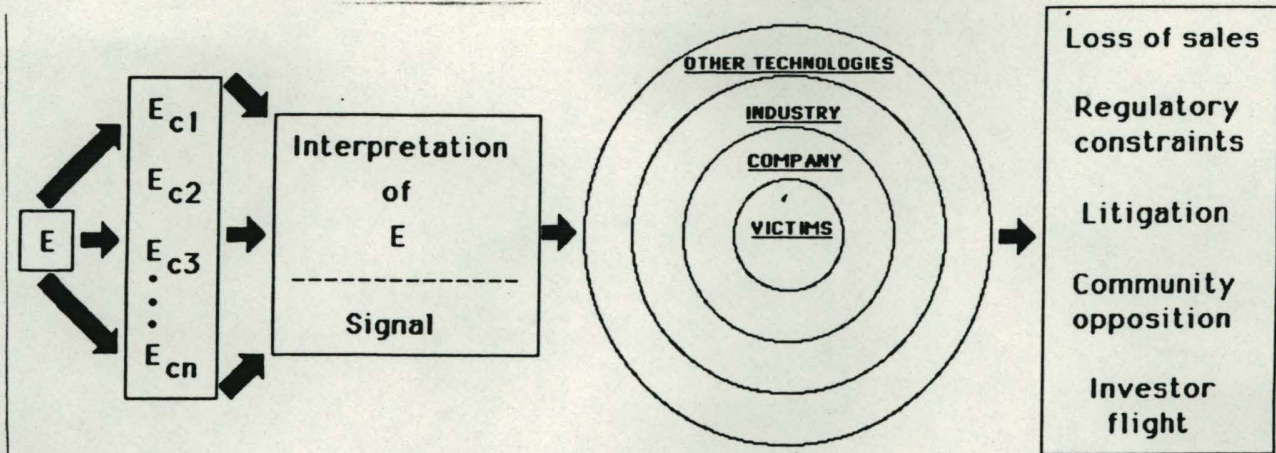

Event Event Interpretation Characteristics

Spread of Impact

Type of Impact (company level)

pacts that might affect corporations and industries. We begin with an Event E, which could be a threatening sign (a defective component, discovery of pollution) or accident in a particular hazard domain. This event can be characterized on a number of dimensions $\mathrm{E}_{\mathrm{c} 1}, \mathrm{E}_{\mathrm{c} 2}, \ldots, \mathrm{E}_{\mathrm{CN}}$ that are presumed to determine the interpretation that people give to that event (e.g., its signal value, seriousness, etc.). This interpretation will determine the event's spread of impact, from immediate victims to the effects on the company and, in extreme cases, to effects on the industry and beyond. The impacts themselves are multidimensional, including loss of sales, new regulatory constraints, litigation, community opposition, and loss of investor confidence. Our objective is to elaborate this framework by specifying, for a broad range of events, the relevant characteristics and the factors that affect their interpretation and impact.

A more complete framework would incorporate the role of intervening variables likely to mediate the relationship between event characteristics and impacts. For example, media response to an event is likely to be a strong determiner of interpretation and impact, as is the response of activist groups such as the Sierra Club or the National Resources Defense Council. Reputation, trust, and management competence are other elements likely to come into play.

In the near term, it is not likely that the monetary costs associated with the impacts in Figure 5 could be forecasted precisely. A more reasonable objective is to identify events that might produce big ripples so that decision makers could consider such warnings in their qualitative deliberations.

One of the first tasks in testing the theory will be to generate a universe of potentially important event characteristics. The literature on perceived risk and signal value suggests the importance of characteristics pertaining to the type of technology (new vs. old), the nature of the effects (contained 
vs. catastrophic; immediate vs. delayed; dreaded or not dreaded), and the nature of the risk (voluntary vs. involuntary; known vs. unknown).

We shall rely heavily on analysis of case histories of unfortunate past events to generate additional ideas about relevant characteristics and their relationship to other variables in the theory. We have assembled a candidate set of about 100 diverse events, including production or manufacturing defects (e.g., botulism in Bon Vivant Soup), discoveries of pollution (e.g., spills of dioxin and PCBs, EDB in grain), chronic health hazards (asbestos, TRIS in pajamas, Thalidomide), acute health hazards (Dalkon Shield IUDs, Legionnaire's disease), external threats such as sabotage or terrrorism (Tylenol tampering, skyjacking), transportation accidents (collision of the two 747's at Tenerife, DC-10), construction flaws (the Hyatt Regency skywalk collapse), and accidents in space technology (Skylab, the shuttle Challenger). Preliminary examination suggests that the existence of identifiable or well-known victims, evidence of criminal behavior or coverup, and evidence of prior warnings will be important event characteristics emerging from these case studies. The goal of this research is to generate a large matrix of events and characteristics describing these events which can be subjected to psychometric analysis to determine the key factors that predict the nature and magnitude of ripple effects.

\section{Conclusion}

Perceived risk leads to social and economic consequences that form a reality which must be considered in risk management. The approach outlined in this paper should be a valuable supplement to risk analysis or costbenefit analysis, making risk managers aware of, and better able to evaluate, the full costs associated with their decisions. This should enable resources to be allocated more wisely to prevent corporate, industrial, and personal tragedies.

\section{Acknowledgement}

This material is based upon work supported by the National Science Foundation under Grant No. SES-8796182 to Decision Research. Any opinions, findings, conclusions, or recommendations expressed in this publication are those of the author and do not necessarily reflect the views of the Foundation.

\section{References}

EPRI Journal. Assessment: The impact and influence of TMI. EPRI Journal, 1980, 5(5), 24-33.

Evans, N., \& Hope, C. Nuclear Power: Futures, Costs, and Benefits. Cambridge: Cambridge University Press, 1984. 
Ferreira, J., \& Slesin, L. Observations on the social impact of large accidents (Technical Report No. 122). Massachusetts Institute of Technology, Operations Research Center, 1976.

Griesmeyer, J.M. and Okrent, D. "Risk management and decision rules for light water reactors". Risk Analysis, 1981, 1, 121-136.

Heising, C.D. and George, V.P. "Nuclear financial risk: Economy-wide costs of reactor accidents". Energy Policy, 1986, 14, 45-51.

Kraus, N.N. and Slovic, P. "Taxonomic analysis of perceived risk: Modeling individual and group perceptions within homogeneous hazard domains". Risk Analysis, in press.

Mishan, E.J. Cost benefit analysis. London: George Allen \& Unwin, 1971.

President's Commission. The accident at Three Mile Island. Washington, DC: U.S. Government Printing Office, 1979.

Raiffa, H. Decision Analysis. Reading, MA: Addison-Wesley, 1968.

Slovic, P. "Perception of risk". Science, 1987, 236, 280-285.

Slovic, P., Fischhoff, B., and Lichtenstein, S. "Characterizing perceived risk". In R.W. Kates, C. Hohenemser, \& J. Kasperson (Eds.), Perilous Progress: Technology as Hazard (pp. 91-123). Boulder, CO: Westview, 1985.

Slovic, P., Lichtenstein, S., and Fischhoff, B. Modelling the Societal Impact of Fatal Accidents. Management Science, 1984, Vol. 30, 464-474.

Slovic, P., MacGregor, D., \& Kraus, N.N. "Perception of risk from automobile safety defects". Accident Analysis and Prevention, 1987, Vol. 19, 359-373.

Wilson, R. "The costs of safety". New Scientist, 1975, 68, 274-275.

Wilson, R., and Crouch, E.P. Risk/benefit analysis. Cambridge, MA: Ballinger, 1982. 